\title{
Analysis of Finite Arrays of Axially Directed Printed Dipoles on Electrically Large Circular Cylinders
}

\author{
Vakur B. Ertürk, Member, IEEE, Roberto G. Rojas, Fellow, IEEE, and Kit Wing Lee
}

\begin{abstract}
Various arrays consisting of finite number of printed dipoles on electrically large dielectric coated circular cylinders are investigated using a hybrid method of moments/Green's function technique in the spatial domain. This is basically an "element by element" approach in which the mutual coupling between dipoles through space as well as surface waves is incorporated. The efficiency of the method comes from the computation of the Green's function, where three types of spatial domain Green's function representations are used interchangeably, based on their computational efficiency and regions where they remain accurate. Numerical results are presented in the form of array current distributions, active reflection coefficient and far-field pattern to indicate the efficiency and accuracy of the method. Furthermore, these results are compared with similar results obtained from finite arrays of printed dipoles on grounded planar dielectric slabs. It is shown that planar approximations, except for small separations, can not be used due to the mutual coupling between the array elements. Consequently, basic performance metrics of printed dipole arrays on coated cylinders show significant discrepancies when compared to their planar counterparts.
\end{abstract}

Index Terms-Coated cylinders, conformal arrays, Green's function, method of moments (MoM), mutual coupling.

\section{INTRODUCTION}

$\mathbf{T}$ HE design and analysis of conformal arrays of printed antennas is of interest in applications ranging from satellite and wireless communications (mobile phone base stations, space division multiple access (SDMA) applications, etc.) to military systems (flush-mounted antennas for aircraft and missiles, radio guidance of missiles, etc.). In these applications, conformality is required for aerodynamic reasons, such that the array elements must conform to their supporting surface, or to reduce the array's radar cross section and in commercial applications for esthetic reasons. However, the lack of adequate design and analysis tools, in particular for electrically large arrays, is a problem that remains to be solved for conformal printed antenna arrays.

Several design tools and numerical techniques have been implemented in CAD packages for the efficient analysis and design of printed finite and infinite arrays for planar geometries [1]-[3]

Manuscript received January 6, 2003; revised August 7, 2003.

V. B. Ertürk is with the Department of Electrical and Electronics Engineering, Bilkent University, TR-06800, Bilkent, Ankara, Turkey (e-mail: vakur@ee.bilkent.edu.tr).

R. G. Rojas is with the Department of Electrical and Computer Engineering, ElectroScience Laboratory, The Ohio State University, Columbus, $\mathrm{OH} 43212$ 1191 USA (e-mail: rojas-teran.1@osu.edu).

K. W. Lee was with the Department of Electrical Engineering, ElectroScience Laboratory, The Ohio State University, Columbus, OH 43212-1191 USA. He is now with Texas Instruments, Inc., Dallas, TX 75243 USA (e-mail: s-lee12@ti.com)

Digital Object Identifier 10.1109/TAP.2004.834443
Note that the analysis of electrically large planar finite arrays is still difficult and it is being investigated by many researchers. In contrast, a limited number of design and numerical analysis tools have been developed for conformal arrays. Unfortunately, the majority of them become inefficient and intractable when the array and/or supporting structure become large. Therefore, one of the most urgent challenges is to develop efficient and accurate design and analysis tools for conformal arrays so that their properties can be accurately predicted, resulting in better designs for the aforementioned applications.

Several authors [4]-[14] have studied the properties of single antennas or arrays of printed antennas on material coated cylinders. However, either some approximate models have been used, where the mutual coupling between the array elements is ignored and, in general, the main focus has been the radiation patterns rather than the currents, or spectral domain techniques have been used, which are applicable to small arrays on electrically small cylinders. Furthermore, for antennas on electrically large cylinders (usually radius is greater than $1 \lambda_{0}$, where $\lambda_{0}$ is the free-space wavelength), the shape of the host cylinder has been considered only for the radiation patterns, and methods applicable to planar geometries have been used in characterizing the input impedance and current distribution since a microstrip antenna is a highly resonant structure [12], [15], [16]. Although this is a reasonable approximation for the case of a single printed antenna, it does not hold for large arrays of printed antennas on large cylinders due to mutual coupling between elements through space and surface waves.

In this paper, our goal is twofold: 1) To perform a full wave analysis of large phased arrays of printed dipoles on electrically large dielectric coated circular cylinders. Note that, to the best of our knowledge, this is the first paper which presents a rigorous analysis of finite phased arrays of printed dipoles on large coated cylinders, where all the mutual coupling effects are incorporated. 2) To make a one-to-one comparison between arrays of printed dipoles on electrically large dielectric coated circular cylinders and arrays of printed dipoles on grounded planar dielectric slabs in terms of currents, reflection coefficient magnitudes and radiation patterns. Thereby showing some interesting features of arrays of printed dipoles on cylindrical structures which are different from their planar counterparts. To achieve these goals, various arrays consisting of finite number of printed dipoles on electrically large dielectric coated circular cylinders are investigated using an efficient and accurate hybrid method based on the combination of method of moments (MoM) with an appropriate spatial domain cylindrical Green's function. This method is basically an "element by element" approach in which the mutual coupling between the dipoles is incorporated. 
Although this calculation may appear to be extremely time consuming for large arrays, it is efficient for large cylinders because of the fast computation of the Green's function which is the kernel of the integral equation to be solved via MoM. Therefore, based on their computational efficiency and accuracy, three types of spatial domain cylindrical Green's function representations are used [17]-[20]. Each representation is valid in some region of space and fortunately the three regions overlap, making the transition from one representation to another stable. In Section II, the formulation of the problem, in particular, the integral equation, the MoM procedure and the types of the Green's function representations used in this paper, are presented. In Section III, numerical results are presented in the form of array current distribution, active reflection coefficient magnitude and radiation patterns to assess the accuracy of this method. These results are then compared with those of phased arrays of printed dipoles on grounded planar dielectric slabs. An $e^{j \omega t}$ time dependence is assumed and suppressed throughout this paper.

\section{FORMULATION}

Fig. 1(a) shows the geometry of a finite, periodic array of $(2 N+1) \times(2 M+1)$ identical printed dipoles mounted on the outer surface of a cylindrically shaped dielectric substrate with an inner radius $a$, outer radius $d$, thickness $d-a=t_{h}$ and relative permittivity $\epsilon_{r}>1$. The substrate is backed by a perfectly conducting, cylindrically shaped ground plane. Both the ground plane and circular cylinder are assumed to be infinite in the $z$ direction. The dipoles are oriented along the $z$ direction and are assumed to be center-fed with infinitesimal generators with impedance $Z_{T}$ as shown in Fig. 1(b). Each dipole is assumed to have a length $L$ and width $W\left[L=2 z_{a}, W=2 r l_{a}\right.$, see Fig. 1(c)] and uniformly spaced from its neighbors by distances $d_{z}$ and $d_{r l}=d \Delta \phi$ in the $z$ and $r l$ directions $(r l=$ $d \phi)$, respectively. Similarly, a finite, planar, periodic array of $(2 N+1) \times(2 M+1)$ identical printed dipoles is shown in Fig. 2. For comparison purposes, the thickness $\left(t_{h}\right)$ and $\epsilon_{r}$ of the substrate, as well as, the feeding mechanism [see Fig. 1(b)] and the size of the dipoles [see Fig. 1(c)] are kept the same. Finally, each dipole on the planar substrate is uniformly spaced from its neighbors by distances $d_{x}$ and $d_{y}$ in the $x$ and $y$ directions, respectively.

\section{A. The MoM Solution}

Similar to [2], the dipoles are assumed to be thin $(W \ll L)$, so only $z$-directed currents are required. Furthermore, only one basis function per dipole is used. Although additional basis functions can be used $(3,5, \ldots)$, the accuracy with one basis function per dipole is good as demonstrated in [2] and [21]. Therefore, the surface current distribution on each dipole is given by

$$
J_{n m}^{s}\left(z^{\prime}, r l^{\prime}\right)=I_{n m} P_{n m}\left(z^{\prime}, r l^{\prime}\right) f_{n m}\left(z^{\prime}, r l^{\prime}\right)
$$

where $I_{n m}$ is the unknown coefficient that determines the total current at the feed point on the element. $f_{n m}\left(z^{\prime}, r l^{\prime}\right)$ on the

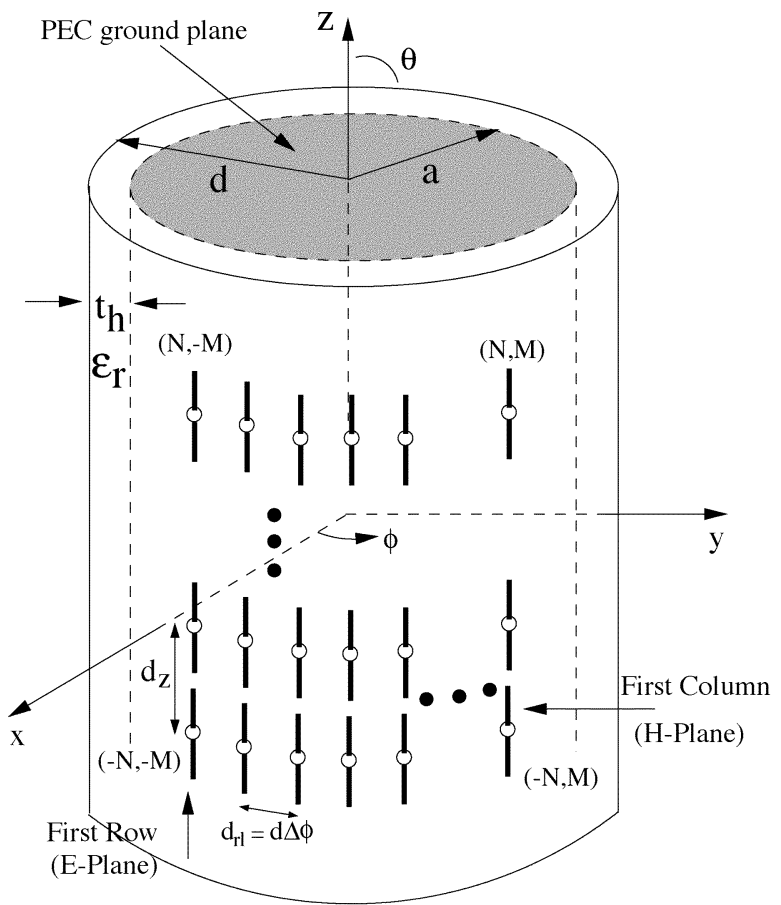

(a)

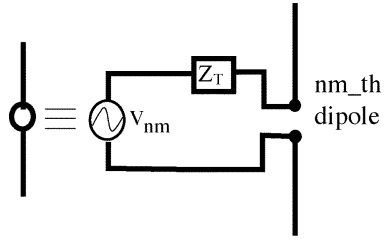

(b)

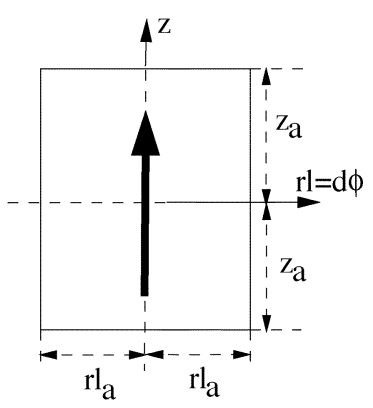

(c)
Fig. 1. (a) Geometry of a periodic array of $(2 N+1) \times(2 M+1)$ printed dipoles on a dielectric coated, electrically large circular cylinder. (b) Dipole connected to an infinitesimal generator with a voltage $V_{n m}$ and a terminating impedance $Z_{T}$. (c) Representation of each dipole for the Method of Moments (MoM) calculations.

$n m$ th $(-N \leq n \leq N,-M \leq m \leq M)$ dipole is expanded in terms of piecewise sinusoidal (PWS) modes defined as

$$
f_{n m}\left(z^{\prime}, r l^{\prime}\right)=\frac{\sin \left[k_{a}\left(\frac{L}{2}-\left|z^{\prime}-n d_{z}\right|\right)\right]}{W \sin \left(k_{a} \frac{L}{2}\right)}
$$

where the wave number $k_{a}$ of the expansion mode has been selected as

$$
k_{a}=k_{0} \sqrt{\frac{\left(\epsilon_{r}+1\right)}{2}}
$$

with $k_{0}$ being the free-space wave number. Note that one can use the effective dielectric constant in (3), namely

$$
k_{a}=k_{0} \sqrt{\epsilon_{e}}
$$

with $\epsilon_{e}$ is given by [21]

$$
\epsilon_{e}=\frac{\epsilon_{r}+1}{2}+\frac{\epsilon_{r}-1}{2}\left(1+\frac{10 t_{h}}{W}\right)^{-\frac{1}{2}},
$$




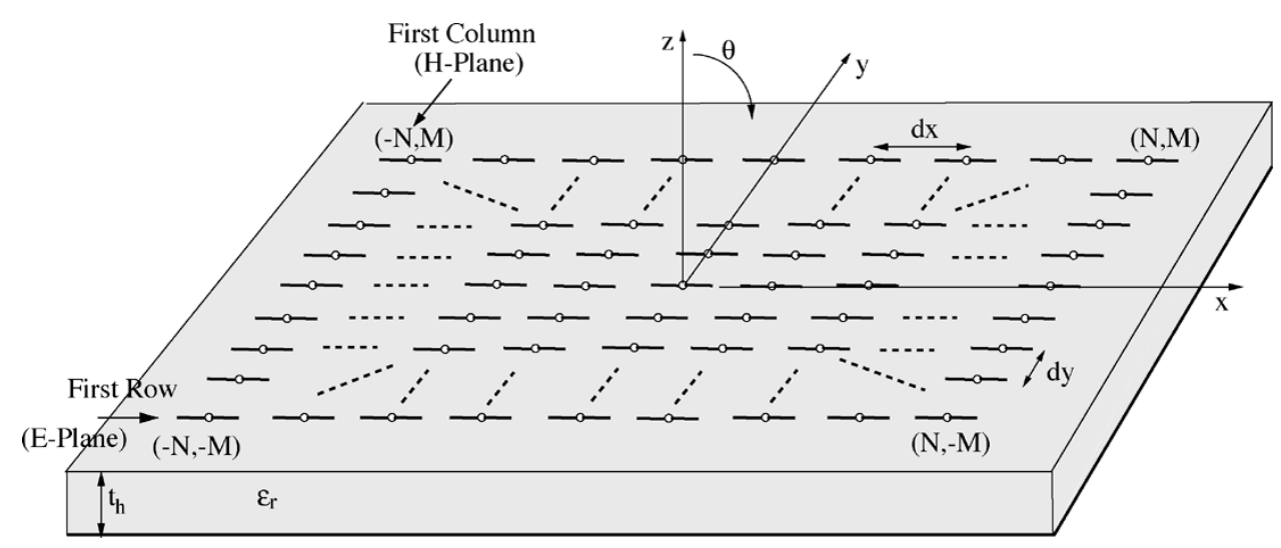

Fig. 2. Geometry of a periodic, planar array of $(2 N+1) \times(2 M+1)$ printed dipoles.

to try to improve the convergence of the MoM solution. However, both choices of $k_{a}$ have yielded the same results in this study, and hence, (3) is chosen in the MoM solution as the wave number of the expansion mode. Finally

$$
P_{n m}\left(z^{\prime}, r l^{\prime}\right)= \begin{cases}1, & z^{\prime}, r l^{\prime} \in n m \text { th element } \\ 0, & \text { otherwise. }\end{cases}
$$

An electric field integral equation (EFIE) is formed by enforcing the boundary condition that the total $E_{z}$ field must vanish on the dipole surfaces. This EFIE, solved using a Galerkin MoM solution and assuming an ideal delta gap generator at the terminals of each center-fed dipole, leads to the following matrix equation [2]:

$$
\left([\mathbf{Z}]+\left[\mathbf{Z}_{\mathbf{T}}\right]\right) \cdot \mathbf{I}=\mathbf{V}
$$

which is alternatively expressed as

$$
\begin{aligned}
& \sum_{n=-N}^{N} \sum_{m=-M}^{M} I_{n m}\left(Z_{n m, p q}+Z_{T_{n m, p q}} \delta_{n m, p q}\right) \\
& \quad=V_{p q}=e^{-j k_{0} \sin \theta_{i} d \cos \left(\phi_{i}-p \Delta \phi\right)} e^{-j k_{0} \cos \theta_{i} q d_{z}}
\end{aligned}
$$

allowing $p$ and $q$ to take on values $-N \leq p \leq N$, and $-M \leq$ $q \leq M$ and

$$
\delta_{n m, p q}= \begin{cases}1, & n m=p q \\ 0, & \text { otherwise }\end{cases}
$$

In (8), the right-hand side, $V_{p q}$, is the voltage excitation which is applied to the terminals of the $p q$ th center-fed dipole antenna element, and is obtained using one expansion mode per dipole with $\left(\theta_{i}, \phi_{i}\right)$ being the scan direction of the beam. On the other hand, $[\mathbf{Z}]$ on the left-hand side of (7) is the impedance matrix of the array with elements $Z_{n m, p q}$, given by

$Z_{n m, p q}=\int_{S_{p q}} d s_{p q} \int_{S_{n m}} d s_{n m}^{\prime} f_{p q}\left(\mathbf{r}_{p q}\right) G_{z z}\left(\mathbf{r}_{p q} / \mathbf{r}_{n m}^{\prime}\right) f_{n m}\left(\mathbf{r}_{n m}^{\prime}\right)$

where $\mathbf{r}_{p q}$ and $\mathbf{r}_{n m}^{\prime}$ are the position vectors of the $p q$ th and $n m$ th dipoles, and $G_{z z}\left(\mathbf{r}_{p q} / \mathbf{r}_{n m}^{\prime}\right)$ is the corresponding component of the appropriate dyadic Green's function for a dielectric coated, electrically large circular cylinder [17]-[20]. Finally, $\left[\mathbf{Z}_{\mathbf{T}}\right]$ in (7)

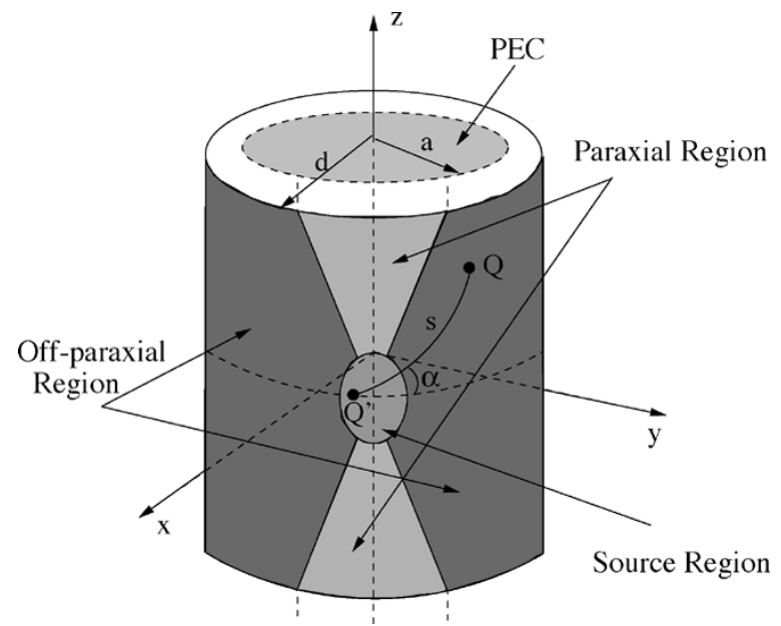

Fig. 3. Regions where each of the three different spatial domain Green's function representations are accurate.

is the generator terminating impedance matrix [which is a diagonal matrix, whose elements are represented by $Z_{T_{n m, p q}} \delta_{n m, p q}$ in (8) with $\delta_{n m, p q}$ defined in (9)], and $\mathbf{I}=\left[I_{n m}\right]$ is the unknown vector of expansion coefficients. In all the examples shown in this paper, all the array elements are excited and therefore the matrix $\left[\mathbf{Z}_{\mathbf{T}}\right]$ is zero. However, if we were to calculate the active element pattern where only one element is excited, the matrix $\left[\mathbf{Z}_{\mathbf{T}}\right]$ would not be zero, and the value of each entry would be equal to the conjugate of the isolated dipole input impedance [2].

\section{B. Computational Efficiency and Green's Function}

Because of the large number of printed dipoles mounted on the electrically large dielectric coated circular cylinder, special attention must be given to the efficient calculation of the impedance matrix. The computational efficiency of this method is strongly dependent on the calculation of the appropriate Green's function component $G_{z z}\left(\mathbf{r}_{p q} / \mathbf{r}_{n m}^{\prime}\right)$ for arbitrary source and observation locations. Therefore, $G_{z z}\left(\mathbf{r}_{p q} / \mathbf{r}_{n m}^{\prime}\right)$ is efficiently and accurately calculated using three different types of spatial domain representations, each valid and accurate in a different region of space on the cylinder as illustrated in Fig. 3.

The first one is a steepest descent path (SDP) representation of the dyadic Green's function [17], which is valid on the 


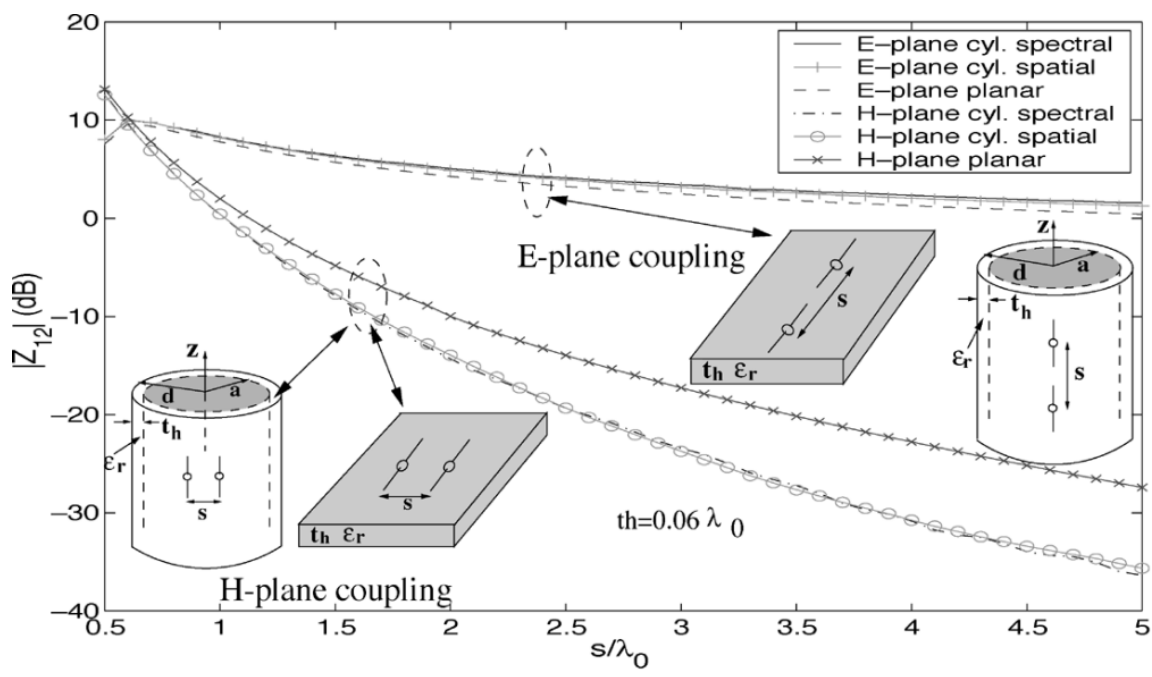

(a)
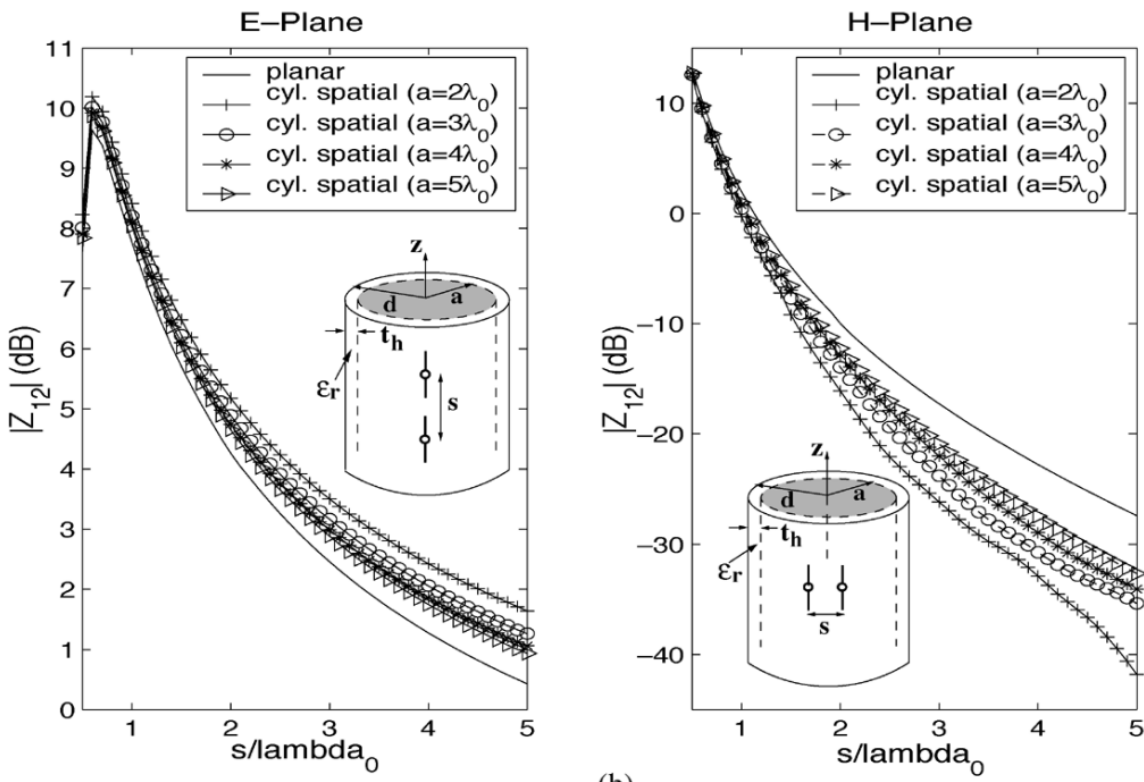

(b)

Fig. 4. Magnitude of the mutual coupling $Z_{12}$ versus separation $s$ between two identical printed dipole modes along the $\mathrm{E}$ and $\mathrm{H}$ planes for a coated cylinder with $\epsilon_{r}=3.25$ and $t_{h}=0.06 \lambda_{0}$. The dimension of each dipole is $(L, W)=\left(0.39 \lambda_{0}, 0.01 \lambda_{0}\right)$. The radii of the cylinders are (a) $a=3 \lambda_{0},(\mathrm{~b}) a=2 \lambda_{0}$, $a=3 \lambda_{0}, a=4 \lambda_{0}, a=5 \lambda_{0}$, respectively.

off-paraxial region as depicted in Fig. 3. This representation is based on the efficient numerical evaluation of a circumferentially propagating ( $\phi$-propagating) series representation of the appropriate Green's function along an SDP on which the integrand decays most rapidly. Using this representation, the surface field component in the $z$ direction at $\rho=d$ excited by a $z$-directed source (again at $\rho^{\prime}=d$ ) is given by [17]

$E_{z}(\alpha, s) \approx \frac{\sqrt{2} e^{-\frac{j 3 \pi}{4}}}{4 \pi^{2} d}$
$\times \sum_{q=1}^{Q}\left(\frac{e^{-j k_{0} s}}{\sqrt{k_{0} s-\frac{j t_{q}^{2}}{2}}} \int_{C_{\tau}\left(t_{q}\right)} \tilde{G}_{z z}\left(t_{q}, \tau\right) P_{e}^{z} e^{-j \xi \tau} d \tau\right) w_{q}$

where $s$ is the arc length of the geodesic path on the surface of the coating from the source to the observation locations, $\alpha$ is the angle between the ray path and the circumferential axis, $t_{q}$ are the roots of the Hermite polynomials and $w_{q}$ are the appropriate weights (Gauss-Hermite quadrature) whose values can be found in [22]. The parameter $\xi=\left(k_{t 0} d / 2\right)^{1 / 3}\left(\phi-\phi^{\prime}\right)$, where $k_{t 0}$ is the transverse propagation constant in free-space, evaluated at the saddle point $\xi_{\mathrm{SDP}} / s$ can be used to determine where this representation is valid. For $\xi_{\mathrm{SDP}} / s>0.2$ and terms other than the self-term, this representation is used, which implies the offparaxial region. The definition of the integration contour $C_{\tau}\left(t_{q}\right)$ and the explicit expressions for $\tilde{G}_{z z}\left(t_{q}, \tau\right)$ are given in [17].

The second representation is the paraxial spatial domain representation of the dyadic Green's function [18], which complements the SDP representation along the paraxial (nearly axial) region $\left(\xi_{\mathrm{SDP}} / s \leq 0.2\right.$ and terms other than the self-term) as depicted in Fig. 3. This representation is based on the periodicity of the $\phi$-propagating series representation of the appropriate Green's function in one of its variables. Making use of this periodicity, the Green's function is approximated by a Fourier series 


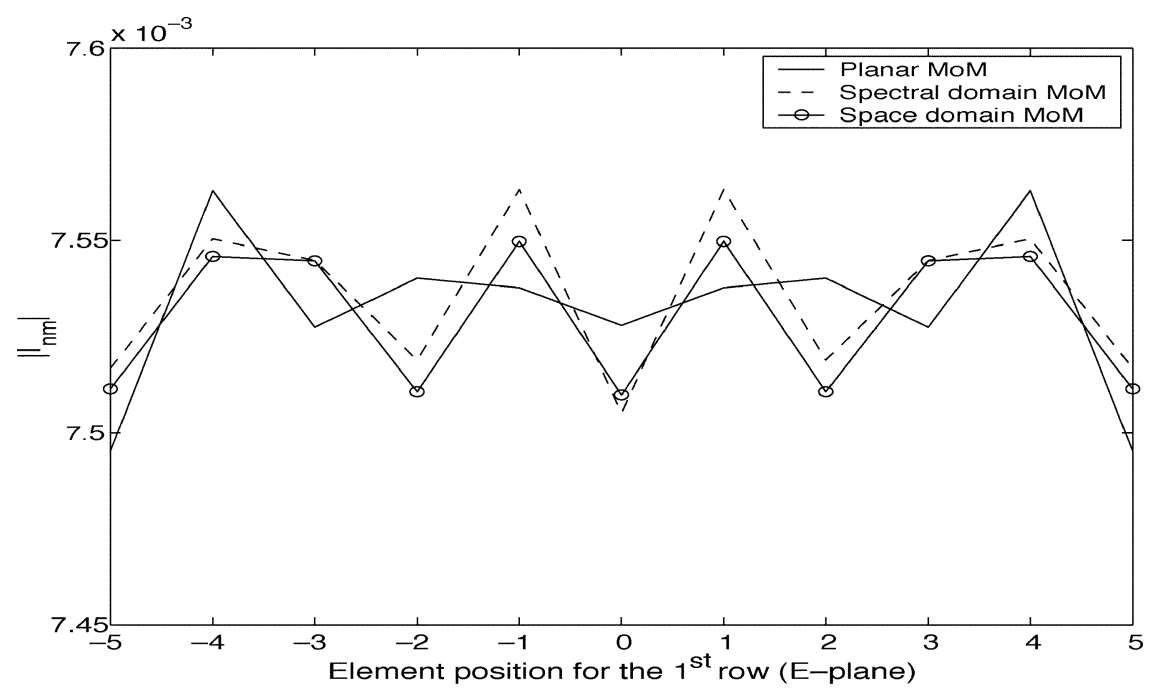

(a)

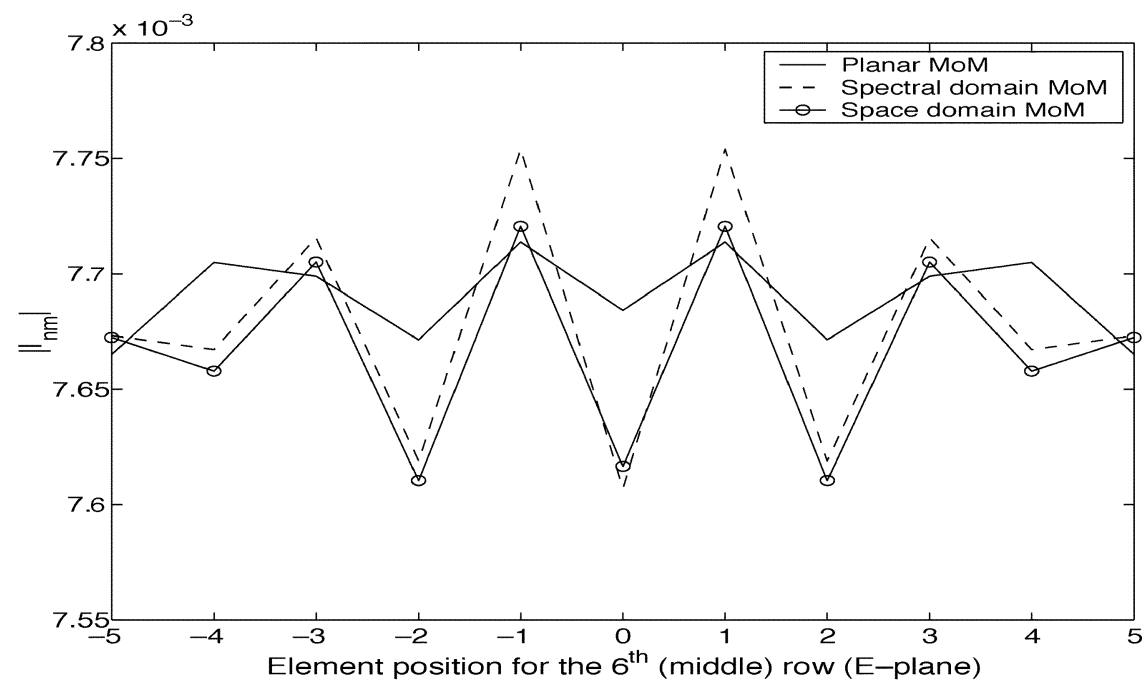

(b)

Fig. 5. Comparison of the magnitude of the induced current $\left|I_{n m}\right|$ obtained via cylindrical spatial, cylindrical spectral and planar MoM for the (a) first row (E plane) $(\mathrm{n}=-5: 5$ and $\mathrm{m}=-5)$; (b) middle (6th) row (E plane) $(\mathrm{n}=-5: 5$ and $\mathrm{m}=0)$ of a $11 \times 11$ printed dipole array. Cylinder parameters: $a=3 \lambda_{0}$, $t_{h}=0.06 \lambda_{0}$ and $\epsilon_{r}=3.25$.

(FS) where the coefficients of this series expansion are easily obtained by a simple numerical integration algorithm. Based on numerical experimentation, including only the two leading terms of the expansion is sufficient in most cases. Furthermore, the accuracy of this representation as well as the ease of its evaluation are determined by the type of algorithm used to calculate the FS coefficients. Using this representation, the $z$ component of the surface field at $\rho=d$ excited by a $z$-directed source at $\rho^{\prime}=d$ is given by [18]

$$
E_{z}(\delta, s) \approx \frac{-Z_{0}}{2 \pi k_{0}}\left\{k_{0}^{2} P(s)+\frac{\partial^{2}}{\partial z^{2}}[P(s)-Q(s)]\right\}
$$

where $P(s)$ and $Q(s)$ are special functions explicitly given in [18]. The partial derivatives with respect to $z$ can be transferred to the basis and testing functions using integration by parts.

Finally, when the distance $s$ between the source and observation points is small (i.e. the source region as shown in Fig. 3), the Green's function for the cylinder is approximated by the
Green's function of a cylinder with an infinite radius (i.e. planar approximations for self-term evaluations, namely, diagonal matrix elements of $[\mathbf{Z}]$ ). This approximation is accurate for small $s$ on electrically large ( $d$ is large) cylinders because the surface can be considered to be locally flat. Therefore, a highly efficient integral representation of the planar microstrip dyadic Green's function derived by Barkeshli et al. [20] is used.

\section{Other Definitions and Far-Field Patterns}

Once the matrix equation (7) is solved for the mode currents, several performance metrics for phased arrays given in [2], [23], and [24] are calculated for a finite phased array of printed dipoles on a coated electrically large circular cylinder and compared with the planar results. Among them, the input impedance at the $n m$ th dipole is computed as

$$
Z_{i n}^{n m}=\frac{V_{n m}}{I_{n m}}
$$




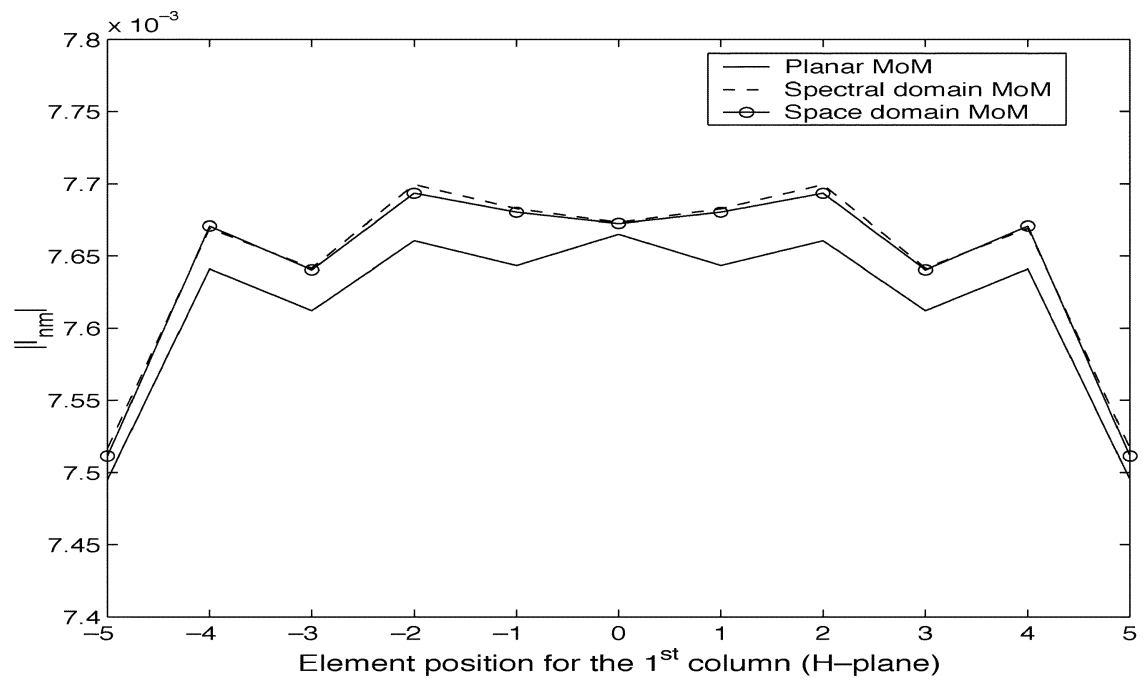

(a)

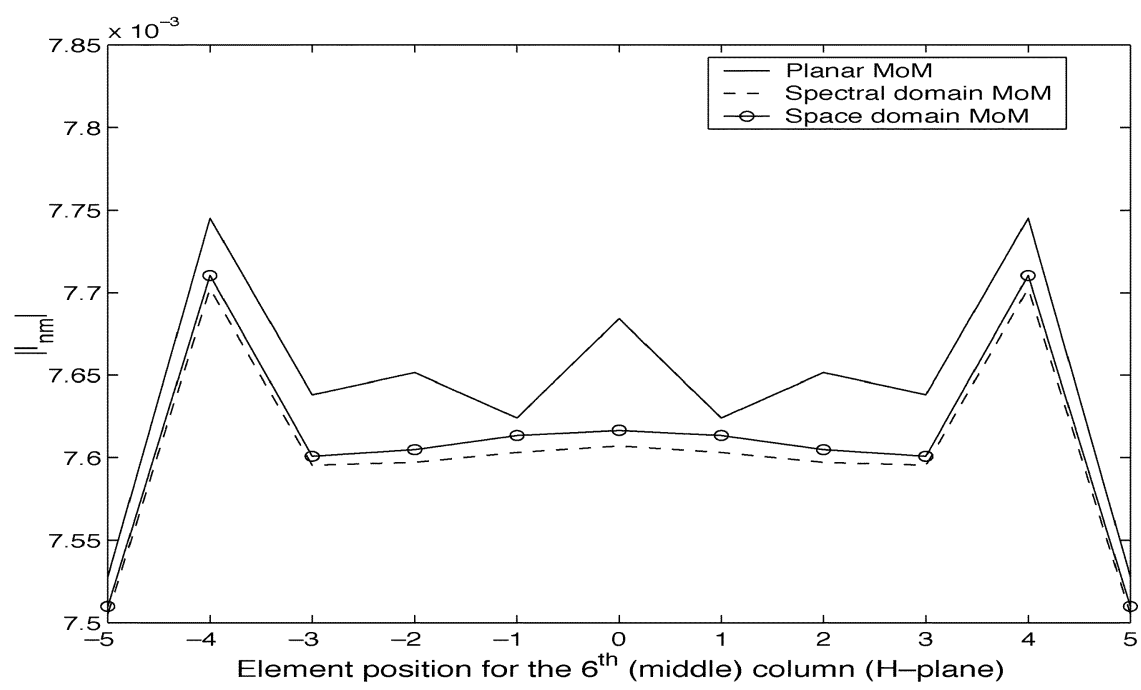

(b)

Fig. 6. Comparison of the magnitude of the induced current $\left|I_{n m}\right|$ obtained via cylindrical spatial, cylindrical spectral and planar MoM for the (a) first column (H plane) $(\mathrm{m}=-5: 5$ and $\mathrm{n}=-5)$; (b) middle (6th) column (H plane) $(\mathrm{m}=-5: 5$ and $\mathrm{n}=0)$ of a $11 \times 11$ printed dipole array. Cylinder same as in Fig. 5 .

and the active reflection coefficient at the $n m$ th dipole is determined by

$$
R^{n m}(\theta, \phi)=\frac{Z_{i n}^{n m}(\theta, \phi)-Z_{i n}^{m i d}\left(\theta=90^{\circ}, \phi=0^{\circ}\right)}{Z_{i n}^{n m}(\theta, \phi)+Z_{i n}^{m i d *}\left(\theta=90^{\circ}, \phi=0^{\circ}\right)}
$$

so that the array is matched to its broadside scan impedance. Here, the superscript 'mid' stands for the middle element of the array.

Finally, for the far-field patterns of the array, initially the far-field element pattern of each dipole on a dielectric coated circular cylinder is calculated either asymptotically as presented in [25] or using a reciprocity approach as presented in [26]. In both solutions, the dipole currents obtained from the solution of (7) are used, and both solutions yield exactly the same result. Once the far-field element pattern of each dipole is determined, the far-field pattern of the array is evaluated using the principle of superposition.

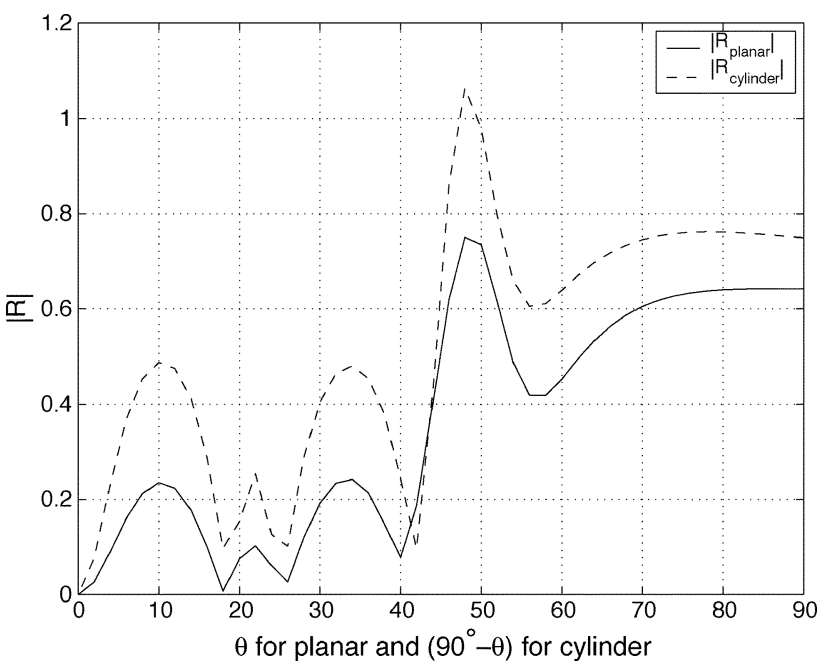

Fig. 7. Magnitude of the reflection coefficient $|R|$ vs. scan angle in the E plane for the center element of an array of $11 \times 11$ printed dipoles on both cylindrical and planar geometries. Cylinder same as in Fig. 5. 
(a)

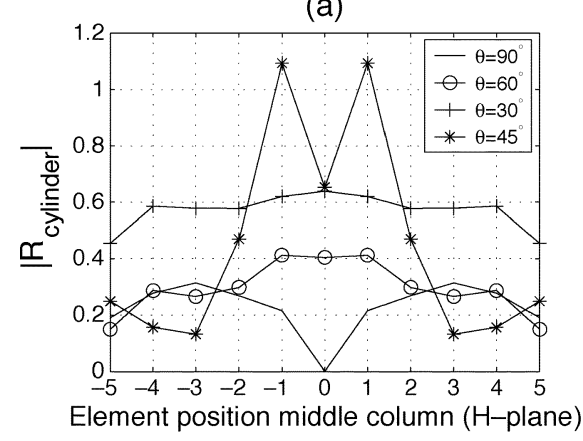

(c)

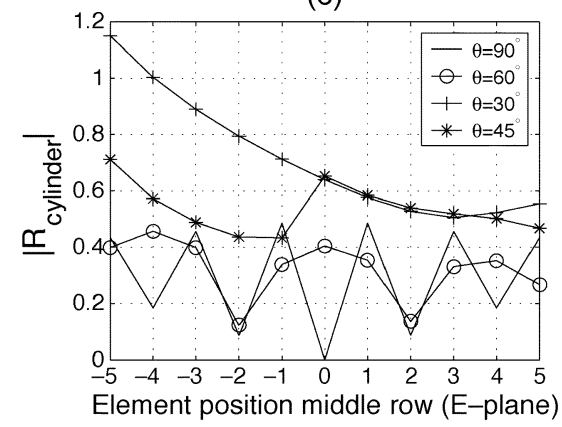

(b)

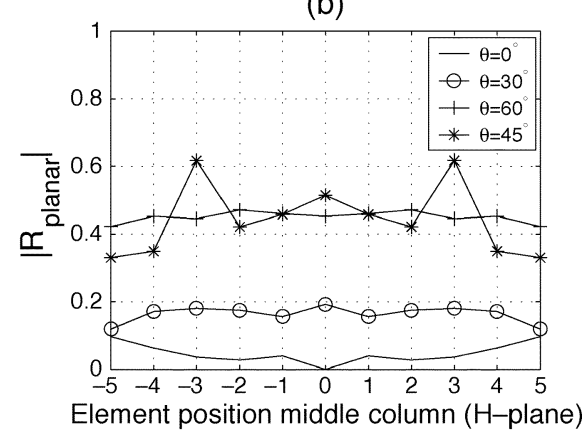

(d)

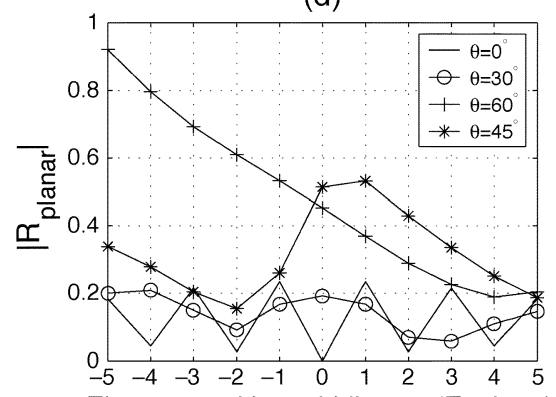

Element position middle row (E-plane)

Fig. 8. Magnitude of the reflection coefficient $|R|$ vs. element position for an array of $11 \times 11$ printed dipoles across the (a) $\mathrm{H}$ plane of the cylindrical geometry $(\mathrm{m}=-5: 5$ and $\mathrm{n}=0)$, (b) H plane of the planar geometry $(\mathrm{m}=-5: 5$ and $\mathrm{n}=0)$, (c) E plane of the cylindrical geometry $(\mathrm{n}=-5: 5$ and $\mathrm{m}=0)$, (d) E plane of the planar geometry $(\mathrm{n}=-5: 5$ and $\mathrm{m}=0)$ for various scan angles. Cylinder same as in Fig. 5.

\section{NUMERICAL RESULTS AND DISCUSSION}

Numerical results are presented: 1) to demonstrate the efficiency and accuracy of the spatial domain MoM/Green's function technique, presented in this paper, for the rigorous analysis of finite phased arrays of printed dipoles on electrically large dielectric coated circular cylinders; 2) to make a one-to-one comparison between arrays of printed dipoles on these cylinders and arrays of printed dipoles on grounded planar dielectric slabs; and 3) to show some interesting properties of arrays of printed dipoles on cylindrical structures which differ from their planar counterparts. To achieve these goals, results obtained with the spatial domain MoM/Green's function technique are compared with the results of an eigenfunction solution based on the spectral domain MoM/Green's function technique for an electrically large dielectric coated circular cylinder with $a=3 \lambda_{0}, t_{h}=0.06 \lambda_{0}$ and $\epsilon_{r}=3.25$ as well as planar results where the same thickness $t_{h}$ and relative dielectric constant $\epsilon_{r}$ are used. In all results, the size of each dipole is selected to be $(L, W)=\left(0.39 \lambda_{0}, 0.01 \lambda_{0}\right)$.

The first numerical result, shown in Fig. 4(a), illustrates the mutual impedance between two identical printed dipole modes [as defined in (10)] versus separation $s$ between the source and observation points along the $\mathrm{E}$ and $\mathrm{H}$ planes, where the cylindrical spatial domain results are compared with the eigenfunction and planar results. The cylindrical results show excellent agreement with each other. Keeping in mind the orientation of the dipoles, the coupling in the E plane of the array (the axial direction of the cylinder) is dominated by surface waves. These surface waves decay at a slower rate than the space waves which are the dominant contributors along the $\mathrm{H}$ plane. Therefore, the coupling along the $\mathrm{E}$ plane is stronger than the coupling along the $\mathrm{H}$ plane as the separation $s$ between the source and observation points increase (except for small separations dominated by the space waves). However, when compared with the planar results, some discrepancies, particularly in the $\mathrm{H}$ plane, are visible. Along the E plane, mutual coupling between two sources on a coated electrically large cylinder resembles to that of a planar case (slightly stronger than the planar case), because the surface waves along the paraxial region of a large cylinder have a behavior that is similar to the surface waves excited on a planar grounded dielectric slab. However, along the $\mathrm{H}$ plane (the circumferential direction), the mutual coupling (as a function of the separation $s$ ) between two sources on a coated electrically large cylinder is much weaker than that of a planar case due to the curvature of the cylinder. Consequently, this example reveals that in a rigorous analysis of a relatively large array of printed dipoles, planar approximations can not be used due to the mutual coupling effects between the array elements. However, as the radius of the cylinder increases, the mutual coupling between two sources approach to the planar case both in the $\mathrm{E}$ and $\mathrm{H}$ planes as shown in Fig. 4(b). Note that along the H plane, the coupling becomes stronger as the radius increases because the effects of the curvature diminish. However, along the E plane (axial direction), as the radius of the cylinder decreases, the coupling deviates from the planar case and becomes stronger. In the limiting case (for cylinders with very small radii), the surface wave propagation will be similar to that of a small-diameter, coated conducting wire (Goubau line) [27].

The next set of numerical examples illustrate the behavior of the array current distribution, active reflection coefficient and radiation pattern pertaining to a $11 \times 11, z$-directed array of thin $\left(W=0.01 \lambda_{0}\right)$, short $\left(L=0.39 \lambda_{0}\right)$ printed dipoles on a cylinder with $a=3 \lambda_{0}, t_{h}=0.06 \lambda_{0}$ and $\epsilon_{r}=3.25$. The array 


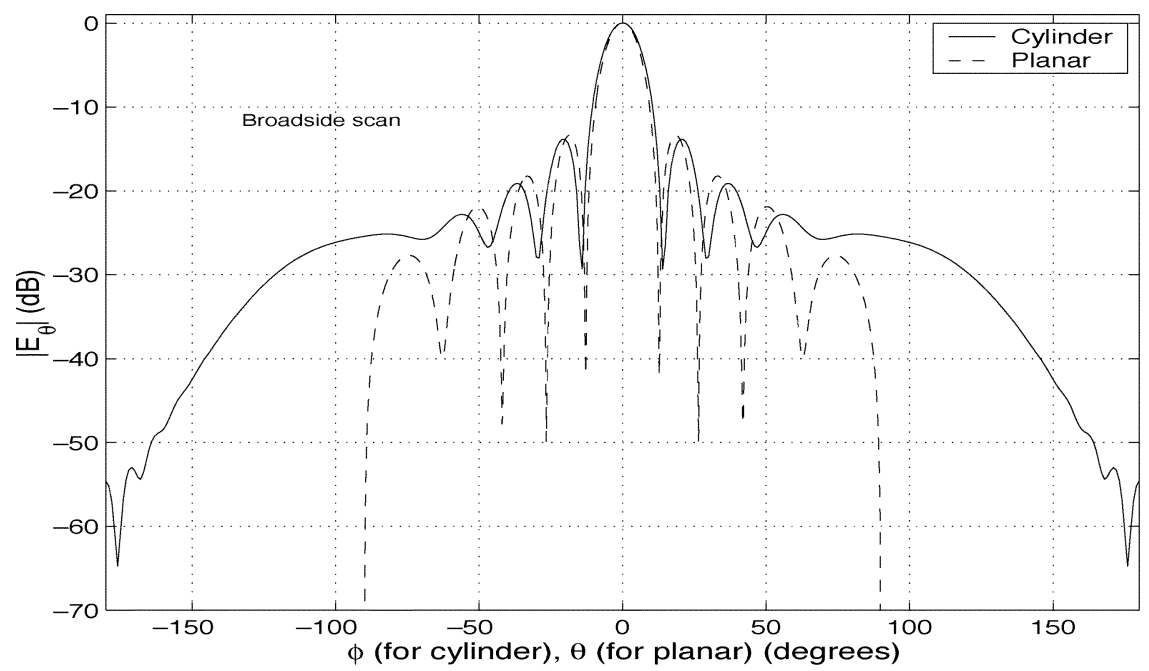

(a)

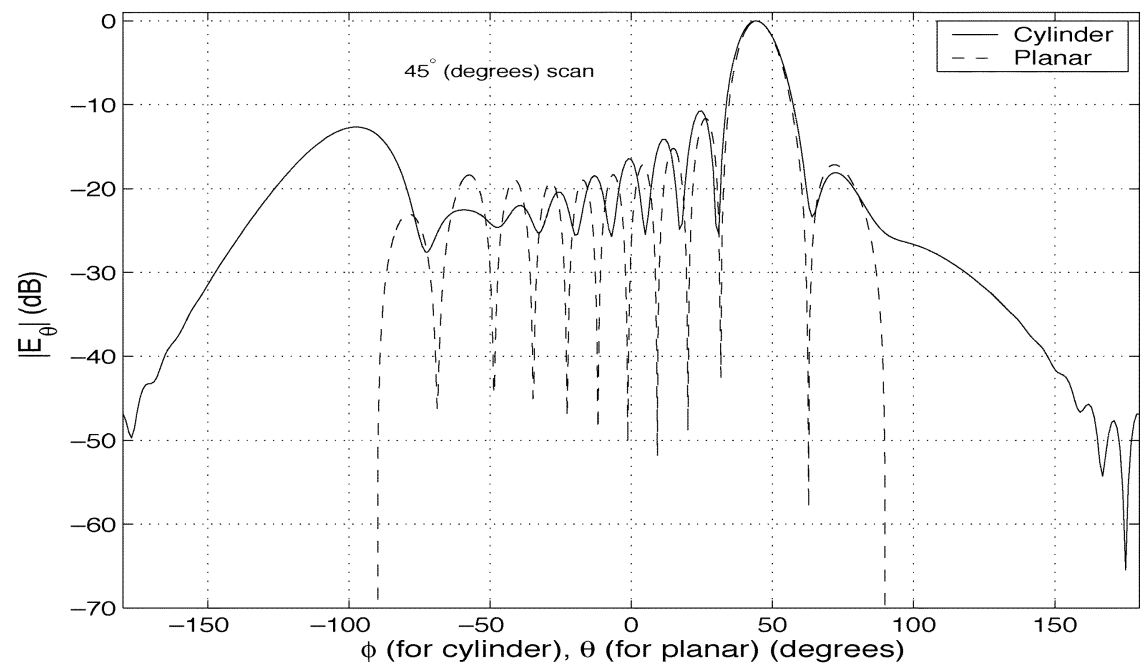

(b)

Fig. 9. Far-field patterns of printed dipole arrays on both the cylinder and planar substrates. All arrays are periodic with $0.5 \lambda_{0}$ in all directions and (a) $9 \times 9$ printed dipole arrays are phased to radiate along the broadside direction, (b) $11 \times 11$ printed dipole arrays are phased to radiate a beam maximum in the direction of $45^{\circ}$. Cylinder same as in Fig. 5.

is periodic with $0.5 \lambda_{0}$ in the $z$ and $r l$ directions, respectively. Similarly, the planar array is also periodic with $0.5 \lambda_{0}$ in the $x$ and $y$ directions, respectively.

Figs. 5 and 6 show the magnitudes of the induced current envelopes (i.e., $\left|I_{n m}\right|$ ) for the first and the middle rows [for Fig. 5(a) and (b), respectively], and the first and the middle columns [for Fig. 6(a) and (b), respectively]. To make a fair comparison between the cylindrical and planar results, the array is excited uniformly and elements of the voltage vector are set to 1 for each of the $p q$ th dipoles. The results obtained with the cylindrical spatial domain results agree well with the reference results (cylindrical spectral domain results). Small disagreements between the spatial and spectral domain results are due to the accuracy problems of the spectral domain results. In a $11 \times 11$ array, the separation between some elements is electrically large. Therefore, mutual impedance calculations between these elements exhibit a convergence problem when the spectral domain technique is used. Although the mutual coupling values are very small between these elements (corresponding entries in the impedance matrix have small values), they still affect the accuracy of the current coefficients $\left(\left|I_{n m}\right|\right)$ and yield a slight disagreement between the spectral and spatial domain results. However, the results for the cylindrical case differ from the planar results (due to the differences in mutual impedances), and such a difference for the array current distribution might be important for some applications.

As seen from the numerical results presented up to this point (Figs. 4-6), results obtained with the spatial domain MoM/Green's function technique agree well with the results obtained with the reference solution (spectral domain). Therefore, for the remaining numerical examples, only the results obtained with the spatial domain MoM/Green's function technique are compared with the planar results, since this technique is more efficient than its spectral domain counterpart due to the efficient evaluation of the Green's function representations. For the cylinder presented in this paper, the computation of SDP representation is approximately 10-11 times faster than the standard spectral domain Green's function representation, 
whereas, the paraxial representation is approximately 5-6 times faster than the standard spectral domain Green's function representation. Also, it should be kept in mind that, as the electrical size (radius) of the cylinder and/or the separation $s$ between the source and observation points get larger, the difference in the computation time for the evaluation of each representation strongly favors the spatial domain representations. Furthermore, for large cylinders and large separations between the source and observation points, the spectral domain solution has severe convergence problems. These are very important issues for the rigorous analysis of large arrays on large cylinders.

Fig. 7 depicts the magnitude of the reflection coefficient versus scan angle of a $11 \times 11$ dipole array on the aforementioned cylinder along the $\mathrm{E}$ plane, and it is compared with the result of a planar array with similar dipoles (and same parameters, i.e. $\epsilon_{r}, t_{h}$, etc.). The array is excited using the right hand side of (8), where $\phi_{i}=0^{\circ}$ and $\theta_{i}$ is varying from $90^{\circ}$ to $0^{\circ}$. A similar excitation is used for the planar array as explained in [2]. Note that in both geometries illustrated in Figs. 1(a) and 2, the angle $\theta$ is defined from the $z$ axis. Therefore, a result obtained at an angle $\theta_{i}$ for the planar case is compared with the result obtained at $90^{\circ}-\theta_{i}$ for the cylinder case. For example, the broadside scan for the planar case occurs at $\left(\theta_{i}, \phi_{i}\right)=\left(0^{\circ}, 0^{\circ}\right)$, whereas, it occurs at $\left(\theta_{i}, \phi_{i}\right)=\left(90^{\circ}, 0^{\circ}\right)$ for the cylindrical geometry. The reflection coefficients of both arrays (cylindrical and planar) are computed at the center element of the arrays which are matched at broadside scan. The result shows that the printed dipole array mounted on the cylinder shows a scan blindness at approximately $\theta_{i}=48^{\circ}$. At this scan angle, the reflection coefficient magnitude of the center element is greater than unity. This simply means that this dipole delivers power to its generator, which is transferred from other ports. Although, the shape of the reflection coefficient magnitude corresponding to the planar case is similar to that of cylinder case and it peaks almost at the same angle, it never exceeds to unity for the parameters used in this example and hence, no scan blindness is observed. Since scan blindness is attributed to the surface wave fields excited within the substrate of this array [1], [2], the curvature of the supporting structure will surely change the behavior of these fields. Therefore, it is not surprising that the results for the planar and cylindrical arrays are somewhat different.

Based on Fig. 7 and as explained in [2], the input impedance across the finite arrays (both cylindrical and planar ones) are expected to be nonuniform. Fig. 8 demonstrates this variation for finite phased arrays of printed dipoles both on grounded cylindrical and planar substrates, showing the magnitude of the reflection coefficient as a function of element position across the $\mathrm{H}$ and $\mathrm{E}$ planes of the $11 \times 11$ array, for various scan angles. In all plots the value of $\phi_{i}=0^{\circ}$. Therefore, a symmetry with respect to the center element is expected and observed across the $\mathrm{H}$ plane as shown in Fig. 8(a) and (b). On the other hand, the $\theta_{i}=90^{\circ}$ (or $\theta_{i}=0^{\circ}$ for planar case) case is symmetric about the center of the array in both planes, and the center element is perfectly matched at broadside, though the other elements are slightly mismatched. For other $\theta_{i}$ values, the mismatch is greater and an asymmetry is observed across the E plane as expected. One can notice that the mismatch for the array of printed dipoles on the cylinder is always greater than its planar counterpart. On the other hand, the $\theta_{i}=45^{\circ}$ data across the $\mathrm{H}$ plane [see Fig. 8(a)] and $\theta_{i}=30^{\circ}$ data across the E plane [see Fig. 8(c)] show that a number of dipole ports on the array have reflection coefficient magnitudes greater than unity, which means that the other dipoles $(|R|<1)$ are absorbing power from their generators and delivering this power to the elements with $|R|>1$. However, for the planar case, although the shape of the reflection coefficient magnitude for each scan angle resembles its cylindrical counterpart, none of them exceeds unity as shown clearly in Fig. 8(b) and (d). Consequently, Figs. 7 and 8 reveal that the curvature plays a significant role for the scan blindness phenomenon for a finite phased array of axially oriented printed dipoles.

Finally, the normalized far-field radiation patterns of a $9 \times 9$ and a $11 \times 11$ phased arrays of printed dipoles on the aforementioned cylinder are obtained using the principle of superposition (i.e. using an element by element approach) and are compared with those of planar ones for two scan angles in Fig. 9(a) and (b), respectively. In both figures, the arrays are scanned along the $\mathrm{H}$ plane. Therefore, for the cylinder geometry, $\theta_{i}$ is kept constant at $90^{\circ}$ while the angle $\phi$ is scanned. For the planar geometry, $\phi_{i}$ is kept at $90^{\circ}$ while the angle $\theta$ is scanned. Also, no special beam forming techniques is applied for the excitation of the arrays. Each dipole is excited uniformly and phased to radiate a beam maximum in the broadside direction and in the direction of $\phi_{i}=45^{\circ}$ for the cylinder $\left(\theta_{i}=45^{\circ}\right.$ for the planar case) in Fig. 9(a) and (b), respectively. Both of the cylindrical printed dipole arrays are periodic with $0.5 \lambda_{0}$ in the $z$ and $r l$ directions. The same periodicity is valid for the planar array in the $x$ and $y$ directions. The size of each dipole as well as the parameters of both the coated cylinder and the grounded slab are kept the same as before. It is observed that pattern of a cylindrical printed dipole array is close to that of a planar one along the main beam and furthermore, the first couple of side lobe levels are also close. However, away from the main lobe, the shape of the patterns change significantly. That is expected and it is due to the curvature of the cylinder. In particular, high sidelobes occur when the cylindrical printed dipole arrays are fed uniformly as illustrated in Fig. 9(b) for the $11 \times 11$ array. This is expected for large conformal arrays since some of the element patterns have their peaks at angles other than the intended main beam direction as explained in [24]. Therefore, the port excitations must be modified for the array on the cylinder to avoid these high sidelobes. In fact, array synthesis algorithms for the cylindrical array have to be different from the well known algorithms for the planar case.

\section{CONCLUSION}

This paper has presented an efficient and accurate analysis of a finite phased array of printed dipoles mounted on an electrically large dielectric coated circular cylinder using a method based on the combination of the MoM with a special Green's function in the spatial domain. The efficiency and accuracy of the method comes directly from the computation of the Green's function, which is the kernel of the EFIE to be solved using the MoM. Therefore, relatively large arrays on electrically large 
coated cylinders can be analyzed rigorously in which the mutual coupling through both the space and surface waves has been incorporated.

Several numerical examples, in the form of mutual coupling between two printed dipole modes and array current distributions for a relatively large array, have been calculated and compared with a reference solution to assess the accuracy of this method. Furthermore, basic performance metrics of arrays have been obtained and compared with similar printed arrays on grounded planar substrates. Certain discrepancies have been observed and discussed.

A number of other related problems, as well as the effect of various array parameters (e.g., element spacing, size of the dipoles, thickness and dielectric constant of the coating, etc.) on the behavior of the array can be addressed to assess the performance of printed phased arrays on electrically large coated cylinders.

\section{REFERENCES}

[1] D. M. Pozar and D. H. Schaubert, "Scan blindness in infinite phased arrays of printed dipoles," IEEE Trans. Antennas Propagat., vol. AP-32, pp. 602-610, June 1984.

[2] D. M. Pozar, "Analysis of finite phased arrays of printed dipoles," IEEE Trans. Antennas Propagat., vol. AP-33, pp. 1045-1053, Oct. 1985.

[3] A. K. Skrivervik and J. R. Mosig, "Finite phased arrays of microstrip patch antennas: the infinite array approach," IEEE Trans. Antennas Propagat., vol. 40, pp. 579-582, May 1992.

[4] A. Nakatini, N. G. Alexopoulus, N. K. Uzunoglu, and P. L. E. Uslenghi, "Accurate Green's function computation for printed circuit antennas on cylindrical antennas," Electromagnetics, vol. 6, pp. 243-254, July-Sept. 1986.

[5] T. M. Habashy, S. M. Ali, and J. A. Kong, "Input impedance and radiation pattern of cylindrical-rectangular and wraparound microstrip antennas," IEEE Trans. Antennas Propagat., vol. 38, pp. 722-731, May 1990.

[6] A. Dreher and M. Thiel, "Dyadic Green's function of multilayer cylindrical closed and sector structures for waveguide, microstrip-antenna, and network analysis," IEEE Trans. Microwave Theory and Techniques, vol. 50, pp. 2576-2579, Nov. 2002

[7] W. Y. Tam, A. K. Y. Lai, and K. M. Luk, "Mutual coupling between cylindrical-rectangular microstrip antennas," IEEE Trans. Antennas Propagat., vol. 43, pp. 897-899, Aug. 1995.

[8] C.-Y. Huang and Y.-T. Chang, "Curvature effects on the mutual coupling of cylindrical-rectangular microstrip antennas," Electron. Lett., vol. 33, pp. 1108-1109, June 1997.

[9] K.-L. Wong, Design of Nonplanar Microstrip Antennas and Transmission Lines. New York: Wiley, 1999.

[10] I. Jayakumar, R. Garg, B. K. Sarap, and B. Lal, "A conformal cylindrical microstrip array for producing omnidirectional radiation pattern," IEEE Trans. Antennas Propagat., vol. 34, pp. 1258-1261, Oct. 1986.

[11] C. M. Da Silva, F. Lumini, J. S. Da S. Lacava, and F. P. Richards, "Analysis of cylindrical arrays of microstrip rectangular patches," Electron. Lett., vol. 27, pp. 778-780, April 1991.

[12] D. I. Wu, "Omnidirectional circularly-polarized microstrip array for telemetry applications," in Proc. IEEE Antennas and Propagat. Symp. Dig., vol. 2, June 1995, pp. 998-1001.

[13] D. Loffler, W. Wiesbeck, and B. Johannisson, "Conformal aperture coupled microstrip phased array on a cylindrical surface," in Proc. IEEE Antennas and Propagat. Symp. Dig., vol. 2, July 1999, pp. 882-885.

[14] Y. Liu, Y. Wang, and R. Yang, "A cylindrical spiral patch array," IEEE Trans. Antennas Propagat., vol. 49, pp. 196-199, Feb. 2001.

[15] K. M. Luk, K. F. Lee, and J. S. Dahele, "Analysis of cylindrical-rectangular patch antenna," IEEE Trans. Antennas Propagat., vol. 37, pp. 143-147, Feb. 1989.

[16] J.-M. Jin, J. A. Berrie, R. Kipp, and S.-W. Lee, "Calculation of radiation patterns of microstrip antennas on cylindrical bodies of arbitrary cross section," IEEE Trans. Antennas Propagat., vol. 45, pp. 126-132, Jan. 1997.
[17] V. B. Ertürk and R. G. Rojas, "Efficient computation of surface fields excited on a dielectric coated circular cylinder," IEEE Trans. Antennas Propagat., vol. 48, pp. 1507-1516, Oct. 2000.

[18] - "Paraxial space-domain formulation for surface fields on dielectric coated circular cylinder," IEEE Trans. Antennas Propagat., vol. 50, pp. 1577-1587, Nov. 2002.

[19] — - "Efficient analysis of input impedance and mutual coupling of microstrip antennas mounted on large coated cylinders," IEEE Trans. Antennas Propagat., vol. 51, pp. 739-749, Apr. 2003.

[20] S. Barkeshli, P. H. Pathak, and M. Marin, "An asymptotic closed-form microstrip surface Green's function for the efficient moment method analysis of mutual coupling in microstrip antennas," IEEE Trans. Antennas Propagat., vol. 38, pp. 1374-1383, Sept. 1990.

[21] D. M. Pozar, "Input impedance and mutual coupling of rectangular microstrip antennas," IEEE Trans. Antennas Propagat., vol. AP-30, pp. 1191-1196, Nov. 1982.

[22] M. Abramowitz and I. A. Stegun, Handbook of Mathematical Functions. New York: Dover Publications, 1970.

[23] R. C. Hansen, Phased Array Antennas. New York: Wiley, 1998.

[24] R. J. Mailloux, Phased Array Antenna Handbook. Boston: Artech, 1994.

[25] J. Ashkenazy, S. Shtrikman, and D. Treves, "Electric surface current model for the analysis of microstrip antennas on cylindrical bodies," IEEE Trans. Antennas Propagat., vol. 33, pp. 295-300, Mar. 1985.

[26] R. A. Martin and D. H. Werner, "A reciprocity approach for calculating the far-field radiation patterns of a center-fed helical microstrip antenna mounted on a dielectric coated circular cylinder," IEEE Trans. Antennas Propagat., vol. 49, pp. 1754-1762, Dec. 2001.

[27] G. Goubau, "Surface waves and their applications to transmission lines," J. Appl. Phys., vol. 21, pp. 1119-1128, Nov. 1950.

Vakur B. Ertürk (M'00) received the B. S. degree in electrical engineering from the Middle East Technical University, Ankara, Turkey, in 1993 and the M. S. and Ph.D. degrees from The Ohio-State University (OSU), Columbus, in 1996 and 2000, respectively.

He is currently an Assistant Professor with the Electrical and Electronics Engineering Department, Bilkent University, Ankara. His research interests include the design and analysis of planar and conformal arrays, active integrated antennas, analysis of propagation over large terrain profiles.

Roberto G. Rojas (S'80-M'85-SM'90-F'01) received the B.S.E.E. degree from New Mexico State University, Las Cruces, in 1979, and the M.S. and $\mathrm{Ph} . \mathrm{D}$. degrees in electrical engineering from The Ohio State University (OSU) Columbus, in 1981 and 1985, respectively.

He is currently a Professor in the Department of Electrical and Computer Engineering with The Ohio State University. His current research interests include the analysis and design of conformal arrays, active integrated arrays, nonlinear microwave circuits, as well as the analysis of electromagnetic radiation and scattering phenomena in complex environments.

Dr. Rojas an elected Member of U.S. Commission B of URSI. He won the 1988 R. W. P. King Prize Paper Award, the 1990 Browder J. Thompson Memorial Prize Award, both given by IEEE, and the 1989 and 1993 Lumley Research Awards, given by the College of Engineering, OSU. He has served as a Chairman, Vice-Chairman, and Secretary/Treasurer of the Columbus, $\mathrm{OH}$ Chapter of the IEEE Antennas and Propagation and Microwave Theory and Techniques Societies.

Kit Wing Lee received the B.S.E.E. and MS. degrees from The Ohio State University, in 1996 and 1998, respectively, where he is currently working toward the Ph.D. degree in the Department of Electrical and Computer Engineering.

Since January of 2003, he has been employed by Texas Instruments, Inc., Dallas, TX, as an IC designer. His research interests include RF and microwave circuit design, computational electromagnetic techniques, coupled oscillator antenna array and ultrawide-band technologies. 